\title{
Effects of sarcopenia, hypoalbuminemia, and laparoscopic surgery on postoperative complications in elderly patients with colorectal cancer: A prospective study
}

\author{
W. S. CHEN ${ }^{1, *}$, Y. S. HUANG ${ }^{1, *}$, L. B. XU' ${ }^{1}$, M. M. SHI', X. D. CHEN ${ }^{2}$, G. Q. YE 2 , T. T. WU ${ }^{3}$, G. B. ZHU ${ }^{1, *}$ \\ ${ }^{1}$ Department of Gastrointestinal Surgery, The First Affiliated Hospital, Wenzhou Medical University, Wenzhou, Zhejiang Province, China; \\ ${ }^{2}$ Department of Gastrointestinal Surgery, The Second Affiliated Hospital, Wenzhou Medical University, Wenzhou, Zhejiang Province, China; \\ ${ }^{3}$ Department of General Surgery, The First Affiliated Hospital of Huzhou University, Huzhou, Zhejiang Province, China
}

${ }^{*}$ Correspondence: $w m c z g b @ 126 . c o m$

${ }^{*}$ Contributed equally to this work.

Received September 8, 2019 / Accepted November 18, 2019

\begin{abstract}
With the increasing number of elderly patients, the risk of diseases such as colorectal cancer (CRC) has increased. The objective of this prospective study was to explore the effects of sarcopenia, hypoalbuminemia, and laparoscopic surgery on postoperative complications among elderly patients who recently underwent colorectal surgery. Patients aged over 65 years who underwent surgery for CRC at the First Affiliated Hospital of Wenzhou Medical University were considered for this study. The demographical and clinical characteristics of these patients, as well as postoperative complications, were prospectively analyzed. The patients were divided into two groups depending on the diagnosis of sarcopenia, and the clinical variables corresponding to the two groups were compared. Further, the risk factors associated with postoperative complications were evaluated using univariate analysis and multivariate logistic regression analysis. A total of 360 patients fulfilled the inclusion criteria. Incidences of postoperative complications in the sarcopenia and non-sarcopenia groups were at $38.3 \%$ and $27.3 \%$, respectively. In addition, sarcopenia $(\mathrm{p}=0.029)$ and hypoalbuminemia $(\mathrm{p}=0.010)$ were identified as independent risk factors, while laparoscopic surgery $(\mathrm{p}=0.023)$ was identified as a protective factor for postoperative complications. However, laparoscopic surgery was a protective factor for postoperative complications in the colon group only $(\mathrm{p}=0.001)$. Sarcopenia and hypoalbuminemia are independent risk factors that influence the probability of developing complications following CRC surgery. Laparoscopic surgery is a protective factor for postoperative complications of CRC patients, particularly colon cancer patients.
\end{abstract}

Key words: colorectal cancer, postoperative complications, old age, sarcopenia, hypoalbuminemia, laparoscopic surgery

Colorectal cancer (CRC) is one of the most frequently encountered malignancies, being the third and second most common disease in terms of incidence (10.2\%) and mortality $(9.2 \%)$, respectively $[1,2]$. Eventually, the number of elderly patients receiving surgical treatment for CRC will increase in China, as surgical resection is the primary treatment for CRC [3].

The postoperative complication rate for $\mathrm{CRC}$ ranges from $18-38 \%$ [4-8]. However, most of the elderly individuals are also associated with other comorbidities, such as pulmonary or cardiovascular diseases, which increase the risk of postoperative complications. Therefore, it is necessary to analyze the risk factors pertaining to postoperative complications among elderly CRC patients.
Typically, favorable prognoses for elderly cancer patients depend on their physical conditions [9], which can be broadly expressed based on their nutrition status. According to a related study, malnutrition is a risk factor affecting the incidence of postoperative complications [10], and sarcopenia is a major indicator of malnutrition [11]. Sarcopenia is defined as the progressive and extensive loss of skeletal muscle mass and muscle function (strength or physical performance) $[12,13]$, and this may increase the incidence of postoperative complications. Moreover, the incidence of sarcopenia increases with age. As reported in some related studies, lower muscle density may also increase the risk of postoperative death and the incidence of complications; thus, it negatively affects the elderly population $[14,15]$. 
Hypoalbuminemia is also a manifestation of poor nutritional status. Its relationship with adverse surgical outcomes has been recognized [16]. When the human body lacks serum albumin, it causes a variety of adverse reactions because serum albumin participates in various physiological mechanisms of the human body. Therefore, in elderly patients with CRC, we consider that hypoalbuminemia may also affect the occurrence of postoperative complications.

From the time when laparoscopy was established as a surgical technique, it is being widely used for many types of surgeries. Compared to conventional surgery, laparoscopic surgery is a minimally invasive procedure that can reduce the probability of developing postoperative complications [17]. Actually, however, elderly patients often exhibit declining cardiopulmonary function and other complications. Moreover, there is a major controversy on whether laparoscopic surgery is suitable for elderly CRC patients. Therefore, this prospective study was conducted to explore the effects of sarcopenia, hypoalbuminemia, and laparoscopic surgery on postoperative complications in elderly CRC patients.

\section{Patients and methods}

Patients. The Gastrointestinal Surgical Department at the First Affiliated Hospital of Wenzhou Medical University (Wenzhou) recruited 364 CRC patients from January 2016 to December 2018. All the patients fulfilled the following criteria: $1 / \geq 65$ years of age [18]; 2 / definitive diagnosis of CRC; 3/ planning to undergo elective colorectal surgery for CRC; 4/ abdomen computed tomography (CT) performed within two weeks before the surgery in this hospital. Four patients were later excluded because of the following criteria: $1 /$ received preoperative chemotherapy or radiotherapy; 2 / palliative surgery; or 3/ emergency surgery. Thus, in total, 360 patients were selected for our analysis. Laparoscopic surgery was recommended to all patients. However, some people opted for open surgery because they had previous abdominal surgery or did not consent to laparoscopic surgery for financial reasons. All the operations were performed by experienced surgeons, each of whom had worked on over 50 CRC cases. Each patient signed an informed consent form to participate in this study. The study protocol was approved by the Ethics Committee of the First Affiliated Hospital of Wenzhou Medical University (2014 NO.063).

Data extraction. The following parameters were collected from all patients: a/ patient characteristics, including age, sex, body mass index (BMI), American Society of Anesthesiologists (ASA) grade, preoperative hemoglobin concentration (hemoglobin concentration of $<120 \mathrm{~g} / \mathrm{l}$ for males and $<110 \mathrm{~g} / \mathrm{l}$ for females is defined as anemia), preoperative plasma albumin concentration (plasma albumin concentration of $<35 \mathrm{~g} / \mathrm{l}$ is defined as hypoalbuminemia), comorbidity (calculated using the Charlson comorbidity index score), preoperative nutritional risk score (evaluated according to Nutritional Risk Screening (NRS) 2002 [19] within $24 \mathrm{~h}$ after admission where a score of three or more identifies a patient with nutritional risk), total abdominal muscle area (TAMA), L3 skeletal muscle index (L3 SMI), previous abdominal surgery history and tumor-node-metastasis stage of the tumor; b/ operation details, including the resection type (rectum or colon), operation type (laparoscopic surgery or laparotomy), type of anesthesia (epidural anesthesia or general anesthesia), combined resection, and operation time; and c/ postoperative outcomes, including postoperative complications within 30 days after surgery, length of stay, and hospitalization costs.

Postoperative complications were defined as symptoms consistent with the Clavien-Dindo classification [20] criteria and those occurring within 30 days of surgery. The complications were classified by two researchers based on the Clavien-Dindo classification. The number of postoperative complications was defined as Grade II or higher.

Definition of sarcopenia. According to the Asian Working Group for Sarcopenia [13], sarcopenia is defined as low skeletal muscle mass combined with low muscle function (defined by strength or physical performance). The muscle mass can be measured using third lumbar crosssectional CT images [21]. A trained researcher supervised by a senior radiologist manually delineated the organization and analyzed the CT images to measure the TAMA with a dedicated processing system (version 3.0.11.3 BN17 32; INFINITT Healthcare Co., Ltd.), blinded for the patient and surgical features. We used a predetermined Hounsfield unit (HU) threshold for specific tissue boundaries, with HU values from -29 to +150 representing skeletal muscle. The TAMA was normalized to height squared $\left(\mathrm{m}^{2}\right)$ and reported as the $\mathrm{L} 3$ skeletal muscle index $\left(\mathrm{cm}^{2} / \mathrm{m}^{2}\right)$.

The muscle strength and physical performance were assessed using the preoperative grip strength and $6 \mathrm{~m}$ usual gait speed, respectively [13]. All the patients used their dominant hands to squeeze an electronic hand dynamometer (EH101; Zhongshan Camry Electronic Co. Ltd., Guangdong Province, China). Moreover, they were asked to cross a distance of $6 \mathrm{~m}$ at their usual gait speed, and the duration from the first step to the last step over was recorded. Both the tests were conducted within $7 \mathrm{~d}$ prior to surgery, and the maximal values obtained from three consecutive tests were recorded [22].

According to racial specificity, the patients were considered sarcopenic when they showed the following conditions: 1 / low muscle mass (L3 skeletal muscle index of $\leq 40.8 \mathrm{~cm} / \mathrm{m}$ for males and $\leq 34.9 \mathrm{~cm} / \mathrm{m}$ for females) [23]; $2 /$ low muscle strength (hand grip strength of $<26 \mathrm{~kg}$ for males and $<18 \mathrm{~kg}$ for females); and 3/ low muscle performance ( $6 \mathrm{~m}$ travel speed of $<0.8 \mathrm{~m} / \mathrm{s}$ ) [13].

Statistical analysis. The Kolmogorov-Smirnov test was conducted to determine the normal distribution of continuous data. Typically, normally distributed continuous data are expressed using their mean and standard deviation values, while continuous data not conforming to a normal distribution are expressed using the median and interquartile range. 
The categorical data were compared using the Pearson's $\chi^{2}$ test or Fisher's exact test. In contrast, non-normally distributed continuous data and ranked data used the MannWhitney $U$ test. Further, clinically relevant parameters were evaluated using the univariate analysis to identify the potential outcome-associated risk factors. Variables with a p-value $<0.10$ in univariate analysis were incorporated into multivariate (logistic regression) analysis. A difference of $p<0.05$ was considered statistically significant. The SPSS software for Windows (version 25.0 IBM; SPSS Inc., Armonk, New York, USA) was employed for all the statistical analyses.

\section{Results}

Patient population and grouping. Table 1 presents the characteristics of the 360 CRC patients included in the study. Overall, there were 214 (59.4\%) male patients. The patients were divided into two groups according to the presence $(n=133)$ or absence of sarcopenia $(n=227)$. The rates of postoperative complications corresponding to the sarcopenia group and non-sarcopenia group were $38.35 \%$ and $27.31 \%$, respectively, and the mean BMI values of the two groups were 21.63 and 23.21 , respectively. Overall, patients with sarcopenia were older $(\mathrm{p}=0.004)$ and had a lower BMI $(\mathrm{p}<0.001)$, more postoperative complications $(\mathrm{p}=0.029)$, longer hospital stays $(\mathrm{p}<0.001)$, and incurred higher hospitalization costs $(\mathrm{p}<0.001)$, compared to the non-sarcopenia patients. There were no significant differences in terms of the NRS, the Charlson comorbidity index, surgery resection type, operation type, or type of anesthesia between the two groups.

Number and frequency of each complication. There were 153 postoperative events involving 113 patients (31.39\%). Among them, there were 51 patients with sarcopenia, including 71 postoperative events. In addition, the incidence of complications in the sarcopenia group was $38.35 \%$. The actual number and frequency of each complication are given in Table 2. Among these complications, infection-related complications were the most frequent, including wound infection, intra-abdominal infection, and pulmonary infection. The second most common event was persistent hypoalbuminemia that required long-term use of concentrated albumin blood product solution (20-25\%) for nutritional support.

Table 1. Demographic and clinical characteristics of patients.

\begin{tabular}{|c|c|c|c|c|}
\hline Factor & $\begin{array}{c}\text { Total } \\
(\mathbf{n}=360)^{a}\end{array}$ & $\begin{array}{c}\text { Sarcopenia } \\
(\mathrm{n}=133)^{\mathrm{a}}\end{array}$ & $\begin{array}{l}\text { No sarcopenia } \\
\quad(n=227)^{a}\end{array}$ & p-value \\
\hline Age, Years ${ }^{++}$ & $72(11.0)$ & $76(11.0)$ & $71(10.0)$ & $0.004^{*}$ \\
\hline $\mathrm{BMI}^{+}$ & $22.63(3.23)$ & $21.63(2.77)$ & $23.21(3.35)$ & $<0.001^{*}$ \\
\hline Albumin, $\mathrm{g} / \mathrm{L}^{++}$ & $36.6(5.48)$ & $36.4(5.35)$ & $36.8(5.90)$ & 0.349 \\
\hline Hemoglobin, g/L $\mathrm{L}^{++}$ & $116.0(32)$ & $114.0(33)$ & $119.0(32)$ & 0.315 \\
\hline Gender & & & & 0.496 \\
\hline Male & $214(59.4 \%)$ & $76(21.1 \%)$ & $138(38.3 \%)$ & \\
\hline Female & $146(40.6 \%)$ & $57(15.8 \%)$ & $89(24.7 \%)$ & \\
\hline ASA grade & & & & $0.003^{*}$ \\
\hline I & $51(14.2 \%)$ & $30(8.3 \%)$ & $21(5.8 \%)$ & \\
\hline II & $248(68.9 \%)$ & $82(22.8 \%)$ & $166(46.1 \%)$ & \\
\hline III & $61(16.9 \%)$ & $21(5.8 \%)$ & $40(11.1 \%)$ & \\
\hline NRS & & & & 0.743 \\
\hline$<3$ & $193(53.6 \%)$ & $73(20.3 \%)$ & $120(33.3 \%)$ & \\
\hline$\geq 3$ & $167(46.4 \%)$ & $60(16.7 \%)$ & $107(29.7 \%)$ & \\
\hline Operating time, $\min ^{++}$ & & & & 0.310 \\
\hline$>210 \mathrm{~min}$ & $240(65)$ & $240(38)$ & $240(65)$ & \\
\hline$\leq 210 \mathrm{~min}$ & $150(58)$ & $150(60)$ & $150(50)$ & \\
\hline Prior abdominal Surgery & & & & 0.158 \\
\hline Yes & $66(18.3 \%)$ & $19(5.3 \%)$ & $47(13.1 \%)$ & \\
\hline No & $294(81.7 \%)$ & $114(31.7)$ & $180(50.0 \%)$ & \\
\hline Charlson comorbidity index & & & & 0.283 \\
\hline 0 & $133(36.9 \%)$ & $56(15.6 \%)$ & $77(21.4 \%)$ & \\
\hline 1 & $151(42.0 \%)$ & $50(13.9 \%)$ & $101(28.1 \%)$ & \\
\hline$\geq 2$ & $76(21.1 \%)$ & $27(7.5 \%)$ & $49(13.6 \%)$ & \\
\hline Tumor location & & & & 0.739 \\
\hline Rectum & $146(40.6 \%)$ & $52(14.4 \%)$ & $94(26.1 \%)$ & \\
\hline Colon & $214(59.4 \%)$ & $81(22.5 \%)$ & $133(36.9 \%)$ & \\
\hline
\end{tabular}


Table 1. Continued ..

\begin{tabular}{|c|c|c|c|c|}
\hline Factor & $\begin{array}{c}\text { Total } \\
(n=360)^{a}\end{array}$ & $\begin{array}{c}\text { Sarcopenia } \\
(n=133)^{a}\end{array}$ & $\begin{array}{c}\text { No sarcopenia } \\
(\mathbf{n}=227)^{a}\end{array}$ & p-value \\
\hline Epidural anesthesia & & & & 1.000 \\
\hline Yes & $253(70.3 \%)$ & $94(26.1 \%)$ & $159(44.2 \%)$ & \\
\hline No & $107(29.7 \%)$ & $39(10.8 \%)$ & $68(18.9 \%)$ & \\
\hline General anesthesia & & & & 0.730 \\
\hline Yes & $348(96.7 \%)$ & $128(35.6 \%)$ & $220(61.1 \%)$ & \\
\hline No & $12(3.3 \%)$ & $5(1.4 \%)$ & $7(1.9 \%)$ & \\
\hline Operation type & & & & 0.490 \\
\hline Laparoscopic surgery & $123(34.2 \%)$ & $42(11.7 \%)$ & $81(22.5 \%)$ & \\
\hline Open surgery & $237(65.8 \%)$ & $91(25.3 \%)$ & $146(40.6 \%)$ & \\
\hline Combined resection & & & & 0.771 \\
\hline Yes & $20(5.6 \%)$ & $8(2.2 \%)$ & $12(3.3 \%)$ & \\
\hline No & $340(94.4 \%)$ & $125(34.7 \%)$ & $215(59.7 \%)$ & \\
\hline TNM stage & & & & 0.429 \\
\hline $1-2$ & $218(60.6 \%)$ & $77(21.4 \%)$ & $141(39.2 \%)$ & \\
\hline $3-4$ & $142(39.4 \%)$ & $56(15.6 \%)$ & $86(23.9 \%)$ & \\
\hline Postoperative complications & & & & $0.029^{*}$ \\
\hline Yes & $113(31.4 \%)$ & $51(14.2 \%)$ & $62(17.2 \%)$ & \\
\hline No & $247(68.6 \%)$ & $82(22.8 \%)$ & $165(45.8 \%)$ & \\
\hline Duration of hospital stay, DAYS ${ }^{++}$ & $19(9)$ & $20.00(10)$ & $19(9)$ & 0.444 \\
\hline Costs, $\mathrm{RMB}^{++}$ & $50956.92(21010.22)$ & $48558.28(22439.86)$ & $52905.64(20385.51)$ & 1.000 \\
\hline Readmissions within 30 days of discharge & $12(3.33 \%)$ & $10(2.78 \%)$ & $2(0.55 \%)$ & 0.371 \\
\hline
\end{tabular}

ASA = American Society of Anesthesiology; BMI = body mass index; TNM = tumor-node-metastasis; NRS = nutritional risk screening

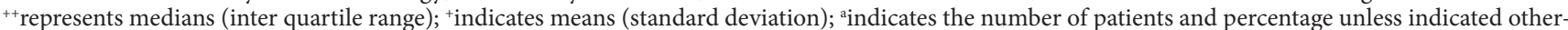
wise; ${ }^{*}$ statistically significant $\mathrm{p}<0.05$

Table 2. Actual number and frequency of each complication.

\begin{tabular}{lccc}
\hline Complicationa & $\begin{array}{c}\text { Total } \\
(\mathbf{n}=113)^{\mathbf{c}}\end{array}$ & $\begin{array}{c}\text { Sarcopenia } \\
\text { group } \\
(\mathbf{n}=5 \mathbf{5 1})^{\mathbf{c}}\end{array}$ & $\begin{array}{c}\text { No sarcopenia } \\
\text { group } \\
(\mathbf{n}=\mathbf{6 2})^{\mathfrak{c}}\end{array}$ \\
\hline Infection-related complications & & & \\
Wound infection & $32(8.89 \%)$ & $14(3.89 \%)$ & $18(5.00 \%)$ \\
Intra-abdominal infection & $21(5.83 \%)$ & $8(2.22 \%)$ & $13(3.61 \%)$ \\
Respiratory tract infection & $18(5.00 \%)$ & $8(2.22 \%)$ & $10(2.78 \%)$ \\
Persistent hypoalbuminemia & $17(4.72 \%)$ & $10(2.78 \%)$ & $7(1.94 \%)$ \\
Anastomotic leakage & $13(3.61 \%)$ & $4(1.11 \%)$ & $9(2.50 \%)$ \\
Venous thrombosis & $11(3.05 \%)$ & $4(1.11 \%)$ & $7(1.94 \%)$ \\
Bowel obstruction & $9(2.50 \%)$ & $3(0.83 \%)$ & $6(1.67 \%)$ \\
Diarrhea & $4(1.11 \%)$ & $2(0.55 \%)$ & $2(0.55 \%)$ \\
Pleural effusion & $4(1.11 \%)$ & $2(0.55 \%)$ & $2(0.55 \%)$ \\
Urinary system & $6(1.67 \%)$ & $4(1.11 \%)$ & $2(0.55 \%)$ \\
Postoperative bleeding & $7(1.94 \%)$ & $5(1.39 \%)$ & $2(0.55 \%)$ \\
Cardiac complications & $2(0.55 \%)$ & $2(0.55 \%)$ & $0(0.00 \%)$ \\
Hepatic complications & $1(0.28 \%)$ & $0(0.00 \%)$ & $1(0.28 \%)$ \\
Others ${ }^{b}$ & $8(2.22 \%)$ & $5(1.39 \%)$ & $3(0.83 \%)$ \\
\hline
\end{tabular}

${ }^{\mathrm{a}} \mathrm{some}$ patients have more than one type of postoperative complication. The total number of postoperative complications is higher than the number of patients who suffer from postoperative complications; ${ }^{\mathrm{b} O t h e r s: ~ r e p r e s e n t ~}$ four severe complications (gastroparesis, lymphorrhagia, nerve problems, and pulmonary embolism) and two mild complications (gout and abdominal distension); ${ }^{c}$ values are the number of patients and percentage of the total number
Risk factors of postoperative complications. Table 3 summarizes the factors related to complications arising after CRC surgery. In the univariate analysis, BMI $(\mathrm{p}=0.092)$, operation type (laparoscopy; $\mathrm{p}=0.023)$, sarcopenia $(\mathrm{p}=0.029)$, and hypoalbuminemia $(\mathrm{p}=0.010)$ were associated with the incidence of complications after CRC surgery. However, no significant differences were observed in terms of the gender, nutritional risk, operation time, anemia, type of resection, ASA grade, and the underlying diseases between the two groups. The results of the multivariate logistic regression analysis adjusted for potential confounders suggested that sarcopenia $(\mathrm{p}=0.045)$ and hypoalbuminemia $(\mathrm{p}=0.022)$ were the undesirable factors that were most markedly correlated with the incidence of complications after CRC surgery. In addition, laparoscopic surgery $(\mathrm{p}=0.042)$ emerged as a protective factor for CRC surgery.

Uni- and multivariate analyses of subgroups. In the subgroup for univariate analysis stratified by the tumor location, there were significant differences in terms of the BMI $(\mathrm{p}=0.020)$ and laparoscopic surgery $(\mathrm{p}=0.001)$ in the colon group (Table 4). Multivariate logistic regression analyses revealed that laparoscopic surgery $(\mathrm{p}=0.002, \mathrm{OR}=0.312)$ was an independent factor affecting postoperative complications. However, in the rectum group (Table 5), we found that laparoscopic surgery $(\mathrm{p}=0.841)$ was not associated with 
Table 3. Univariate and multivariate logistic regression analyses of factors associated with postoperative complications.

\begin{tabular}{|c|c|c|c|c|c|c|}
\hline \multirow[b]{2}{*}{ Factor } & \multicolumn{4}{|c|}{ Univariate analysis } & \multicolumn{2}{|c|}{ Multivariate analysis } \\
\hline & $\begin{array}{c}\text { Total } \\
(n=360)\end{array}$ & $\begin{array}{c}\text { Postoperative } \\
\text { complications }(n=113)\end{array}$ & $\begin{array}{c}\text { Non-postoperative } \\
\text { complications }(n=247)\end{array}$ & p-value & OR $(95 \% \mathrm{CI})$ & p-value \\
\hline Gender & & & & 0.335 & & \\
\hline Male & 214 & 63 & 151 & & & \\
\hline Female & 146 & 50 & 96 & & & \\
\hline ASA grade & & & & 0.638 & & \\
\hline $\mathrm{I}$ & 51 & 18 & 33 & & & \\
\hline II & 248 & 74 & 174 & & & \\
\hline III & 61 & 21 & 40 & & & \\
\hline NRS & & & & 0.297 & & \\
\hline$<3$ & 193 & 56 & 137 & & & \\
\hline$\geq 3$ & 167 & 57 & 110 & & & \\
\hline BMI & & & & 0.092 & & \\
\hline$<18.5$ & 36 & 17 & 19 & & & \\
\hline $18.5-24.0$ & 208 & 63 & 145 & & & \\
\hline$>24.0$ & 116 & 33 & 83 & & & \\
\hline Operating time & & & & 0.290 & & \\
\hline$>210 \mathrm{~min}$ & 87 & 23 & 64 & & & \\
\hline$\leq 210 \mathrm{~min}$ & 273 & 90 & 183 & & & \\
\hline Prior abdominal surgery & & & & 0.884 & & \\
\hline Yes & 66 & 20 & 46 & & & \\
\hline No & 294 & 93 & 201 & & & \\
\hline Charlson comorbidity index & & & & 0.911 & & \\
\hline 0 & 133 & 40 & 93 & & & \\
\hline 1 & 151 & 49 & 102 & & & \\
\hline$\geq 2$ & 76 & 24 & 52 & & & \\
\hline Tumor location & & & & 0.463 & & \\
\hline Rectum & 146 & 49 & 97 & & & \\
\hline Colon & 214 & 64 & 150 & & & \\
\hline Epidural anesthesia & & & & 0.388 & & \\
\hline Yes & 253 & 83 & 170 & & & \\
\hline No & 107 & 30 & 77 & & & \\
\hline General anesthesia & & & & 0.435 & & \\
\hline Yes & 348 & 108 & 240 & & & \\
\hline No & 12 & 5 & 7 & & & \\
\hline Laparoscopy & & & & $0.023^{\star}$ & $0.595(0.360-0.982)$ & $0.042^{*}$ \\
\hline Yes & 123 & 29 & 94 & & & \\
\hline No & 237 & 84 & 153 & & & \\
\hline Combined resection & & & & 0.393 & & \\
\hline Yes & 20 & 8 & 12 & & & \\
\hline No & 340 & 105 & 235 & & & \\
\hline TNM stage & & & & 0.908 & & \\
\hline $1-2$ & 218 & 69 & 149 & & & \\
\hline $3-4$ & 142 & 44 & 98 & & & \\
\hline Sarcopenia & & & & $0.029^{*}$ & $1.606(1.012-2.550)$ & $0.045^{\star}$ \\
\hline Yes & 133 & 51 & 82 & & & \\
\hline No & 227 & 62 & 165 & & & \\
\hline Hypoalbuminemia & & & & $0.010^{*}$ & $1.731(1.082-2.771)$ & $0.022^{\star}$ \\
\hline Yes & 119 & 48 & 71 & & & \\
\hline No & 241 & 65 & 176 & & & \\
\hline Anemia & & & & 0.416 & & \\
\hline Yes & 92 & 32 & 60 & & & \\
\hline No & 268 & 81 & 187 & & & \\
\hline
\end{tabular}

ASA = American Society of Anesthesiology; BMI = body mass index; TNM = tumor-node-metastasis; NRS = nutritional risk screening; ${ }^{\star}$ statistically significant $\mathrm{p}<0.05$ 
Table 4. Univariate and multivariate logistic regression analyses of factors associated with postoperative complications in colon.

\begin{tabular}{|c|c|c|c|c|c|c|}
\hline \multirow[b]{2}{*}{ Factor } & \multicolumn{4}{|c|}{ Univariate analysis } & \multicolumn{2}{|c|}{ Multivariate analysis } \\
\hline & $\begin{array}{c}\text { Total } \\
(\mathrm{n}=214)\end{array}$ & $\begin{array}{c}\text { Postoperative } \\
\text { complications }(n=64)\end{array}$ & $\begin{array}{c}\text { No-postoperative } \\
\text { complications }(n=150)\end{array}$ & p-value & $\begin{array}{c}\text { OR } \\
(95 \% \mathrm{CI})\end{array}$ & p-value \\
\hline Gender & & & & 0.160 & & \\
\hline Male & 116 & 30 & 86 & & & \\
\hline Female & 98 & 34 & 64 & & & \\
\hline ASA grade & & & & 0.773 & & \\
\hline I & 29 & 10 & 19 & & & \\
\hline II & 140 & 42 & 98 & & & \\
\hline III & 45 & 12 & 33 & & & \\
\hline NRS & & & & 0.235 & & \\
\hline$<3$ & 104 & 27 & 77 & & & \\
\hline$\geq 3$ & 110 & 37 & 73 & & & \\
\hline BMI & & & & $0.020^{*}$ & $0.765(0.474-1.232)$ & 0.270 \\
\hline$<18.5$ & 22 & 12 & 10 & & & \\
\hline $18.5-24.0$ & 117 & 29 & 88 & & & \\
\hline$>24.0$ & 75 & 23 & 52 & & & \\
\hline Operating time & & & & 0.466 & & \\
\hline$>210 \mathrm{~min}$ & 44 & 11 & 33 & & & \\
\hline$\leq 210 \mathrm{~min}$ & 170 & 53 & 117 & & & \\
\hline Prior abdominal surgery & & & & 0.715 & & \\
\hline Yes & 45 & 12 & 33 & & & \\
\hline No & 169 & 52 & 117 & & & \\
\hline Charlson comorbidity index & & & & 0.359 & & \\
\hline 0 & 74 & 18 & 56 & & & \\
\hline 1 & 90 & 28 & 62 & & & \\
\hline$\geq 2$ & 50 & 18 & 32 & & & \\
\hline Epidural anesthesia & & & & 0.104 & & \\
\hline Yes & 65 & 14 & 51 & & & \\
\hline No & 149 & 50 & 99 & & & \\
\hline General anesthesia & & & & 0.131 & & \\
\hline Yes & 205 & 59 & 146 & & & \\
\hline No & 9 & 5 & 4 & & & \\
\hline Laparoscopy & & & & $0.001^{*}$ & $0.312(0.151-0.647)$ & $0.002^{*}$ \\
\hline Yes & 71 & 11 & 60 & & & \\
\hline No & 14. & 52 & 90 & & & \\
\hline Combined resection & & & & 0.330 & & \\
\hline Yes & 9 & 4 & 5 & & & \\
\hline No & 205 & 60 & 145 & & & \\
\hline TNM stage & & & & 0.447 & & \\
\hline $1-2$ & 132 & 37 & 95 & & & \\
\hline $3-4$ & 82 & 27 & 55 & & & \\
\hline Sarcopenia & & & & 0.142 & & \\
\hline Yes & 81 & 29 & 52 & & & \\
\hline No & 133 & 35 & 98 & & & \\
\hline Hypoalbuminemia & & & & 0.176 & & \\
\hline Yes & 79 & 28 & 51 & & & \\
\hline No & 135 & 36 & 99 & & & \\
\hline Anemia & & & & 1.000 & & \\
\hline Yes & 72 & 21 & 51 & & & \\
\hline No & 142 & 43 & 99 & & & \\
\hline
\end{tabular}

ASA = American Society of Anesthesiology; BMI = body mass index; TNM = tumor-node-metastasis; NRS = nutritional risk screening; ${ }^{*}$ statistically significant $\mathrm{p}<0.05$ 
Table 5. Univariate and multivariate logistic regression analyses of factors associated with postoperative complications in rectum.

\begin{tabular}{|c|c|c|c|c|c|c|}
\hline \multirow[b]{2}{*}{ Factor } & \multicolumn{4}{|c|}{ Univariate analysis } & \multicolumn{2}{|c|}{ Multivariate analysis } \\
\hline & $\begin{array}{c}\text { Total } \\
(\mathrm{n}=146)\end{array}$ & $\begin{array}{c}\text { Postoperative } \\
\text { complications }(n=49)\end{array}$ & $\begin{array}{c}\text { No-postoperative } \\
\text { complications }(n=97)\end{array}$ & p-value & $\begin{array}{c}\text { OR } \\
(95 \% \mathrm{CI})\end{array}$ & p-value \\
\hline Gender & & & & 1.000 & & \\
\hline Male & 98 & 33 & 65 & & & \\
\hline Female & 48 & 16 & 32 & & & \\
\hline ASA grade & & & & 0.104 & & \\
\hline I & 22 & 8 & 14 & & & \\
\hline II & 108 & 32 & 76 & & & \\
\hline III & 16 & 9 & 7 & & & \\
\hline NRS & & & & 0.755 & & \\
\hline$<3$ & 89 & 29 & 60 & & & \\
\hline$\geq 3$ & 57 & 20 & 37 & & & \\
\hline BMI & & & & 0.339 & & \\
\hline$<18.5$ & 14 & 5 & 9 & & & \\
\hline $18.5-24.0$ & 91 & 34 & 57 & & & \\
\hline$>24.0$ & 41 & 10 & 31 & & & \\
\hline Operating time & & & & 0.443 & & \\
\hline$>210 \mathrm{~min}$ & 43 & 12 & 31 & & & \\
\hline$\leq 210 \mathrm{~min}$ & 103 & 43 & 146 & & & \\
\hline Prior abdominal surgery & & & & 0.634 & & \\
\hline Yes & 21 & 8 & 13 & & & \\
\hline No & 125 & 41 & 84 & & & \\
\hline Charlson comorbidity index & & & & 0.434 & & \\
\hline 0 & 59 & 22 & 37 & & & \\
\hline 1 & 61 & 21 & 40 & & & \\
\hline$\geq 2$ & 26 & 6 & 20 & & & \\
\hline Epidural anesthesia & & & & 0.461 & & \\
\hline Yes & 42 & 15 & 26 & & & \\
\hline No & 104 & 33 & 71 & & & \\
\hline General anesthesia & & & & 0.551 & & \\
\hline Yes & 143 & 49 & 94 & & & \\
\hline No & 3 & 0 & 3 & & & \\
\hline Laparoscopy & & & & 0.841 & & \\
\hline Yes & 52 & 18 & 34 & & & \\
\hline No & 94 & 31 & 63 & & & \\
\hline Combined resection & & & & 1.000 & & \\
\hline Yes & 11 & 4 & 7 & & & \\
\hline No & 135 & 45 & 90 & & & \\
\hline TNM stage & & & & 0.290 & & \\
\hline $1-2$ & 60 & 17 & 43 & & & \\
\hline $3-4$ & 86 & 32 & 54 & & & \\
\hline Sarcopenia & & & & 0.096 & $1.575(0.754-3.290)$ & 0.226 \\
\hline Yes & 52 & 22 & 30 & & & \\
\hline No & 94 & 27 & 67 & & & \\
\hline Hypoalbuminemia & & & & $0.010^{*}$ & $2.196(0.996-4.844)$ & 0.051 \\
\hline Yes & 40 & 20 & 20 & & & \\
\hline No & 106 & 29 & 77 & & & \\
\hline Anemia & & & & $0.029^{*}$ & $1970(0.711-5458)$ & 0.192 \\
\hline Yes & 20 & 11 & 9 & & & \\
\hline No & 126 & 38 & 88 & & & \\
\hline
\end{tabular}

ASA = American Society of Anesthesiology; BMI = body mass index; TNM = tumor-node-metastasis; NRS = nutritional risk screening; ${ }^{\star}$ statistically significant $\mathrm{p}<0.05$ 
postoperative complications. There were significant differences in terms of laparoscopic surgery as a factor affecting postoperative complications between the two subgroups.

\section{Discussion}

Sarcopenia is an age-related disease, as skeletal muscle mass and muscle function decline with age [24-26]. Prior research has established that elderly individuals with sarcopenia demonstrate a higher risk for long hospitalization and require long-term care compared to their non-sarcopenic peers [27]. Moreover, the pathogenesis of skeletal muscle depletion or sarcopenia involves multiple factors including altered nutrition status, chronic inflammatory state, changes in the endocrine environment, and so on [28]. In this study, we found that patients with sarcopenia demonstrated a lower BMI. A patient with remarkable weight loss may be linked to increased risks of aggravated primary disease, morbidity, and mortality $[2,29,30]$. Therefore, we believe that elderly CRC patients with sarcopenia have poor short-term outcomes after CRC surgery [15].

Our findings suggest that early actions should be considered for elderly patients with sarcopenia. The most important intervention is muscle exercise. Effective and reliable resistance exercises contribute toward improving their quality of life and outcomes, as they can maintain and enhance muscle mass and increase the protein content in skeletal muscle [31, 32]. Nutritional support is another key factor, as a change in diet may lead to better outcomes [32, 33]. Appropriate drugs can also be used for treating sarcopenia; however, no clinical studies have confirmed this [34]. In this study, we found that patients with postoperative complications had significantly longer hospital stays and incurred high hospitalization costs. This means that poor postoperative prognosis will increase the economic burden on the society and the families of patients. Therefore, more attention is needed on the adjustment of preoperative elderly sarcopenia patients. Through these measures, we can improve the health condition of elderly patients with sarcopenia before surgery, thereby reducing hospitalization time and costs [35].

Hypoalbuminemia is recognized to be associated with poor prognosis of malignancy [36-38]. Similarly, in this study, hypoalbuminemia was an independent risk factor for postoperative complications. Patients with hypoproteinemia have a higher incidence of postoperative complications. However, this effect is not related to age. For elderly patients, aging does not affect the incidence of hypoalbuminemia [39]. This may be because the reduction in food intake can result in hypoalbuminemia [40, 41]. Furthermore, chronic malnutrition resulting from complications can lead to persistent hypoalbuminemia in the elderly after surgery [42]. In contrast, hypoalbuminemia in elderly patients with CRC demonstrates an explicit relationship with inflammation [43]. During the post-operation acute inflammatory period, vascular permeability, and hepatic protein synthesis are weakened by the activation of reactive proteins [16]. In our opinion, it is imperative to improve the nutritional status of patients before surgery. At the same time, we recommend early enteral nutrition and intravenous supplementation of albumin in elderly patients with persistent hypoproteinemia after surgery [44].

In this study, we considered both open and laparoscopic surgeries. Compared to open surgery, we found that patients who underwent laparoscopic surgery experienced fewer postoperative complications. Therefore, we believe that laparoscopic surgery is beneficial for elderly patients. Most people believe that older people with pneumonia or cardiopulmonary complications are not suitable for laparoscopic surgery because a pneumoperitoneum with carbon dioxide can cause adverse pathophysiological changes (such as hypercapnia), reduce venous return, and increase airway pressure [45]. However, age is not a contraindication for laparoscopic surgery [46], as demonstrated by a lower rate of pneumonia and cardiopulmonary complications, as well as quicker recovery and greater scope for self-care, compared to open surgery $[47,48]$. Furthermore, previous research has established that laparoscopic surgery has a protective effect on tumors in patients [49]. It demonstrates advantages in terms of reduced trauma and bleeding, less pain $[50,51]$, earlier restoration of bowel function, and earlier resumption of normal diet [52]. Therefore, laparoscopic surgery produces more favorable results as it reduces the incidence of postoperative complications in elderly patients relative to open surgery $[50,51]$. In addition, for elderly people who need long-duration laparoscopic surgery, we suggest that sufficient preparation and effective management can reduce the risks and result in safer operations [53].

Overall, we found that laparoscopy is a protective factor for the patient. However, based on previous studies, tumors at different sites have different implications for laparoscopy $[54,55]$; therefore, we performed the subgroup analysis. The single most striking observation was that laparoscopic surgery is not a protective factor for rectal cancer, and the effect of laparoscopic surgery on rectal cancer remains controversial. Some related studies have indicated that laparoscopic surgery exhibits a certain protective effect in terms of rectal cancer $[56,57]$; however, our conclusion contradicts this finding. We detected no difference between laparoscopic and open surgeries in terms of postoperative complications for rectal surgery patients. Moreover, other studies have presented observations that support our conclusion [54].

This study suffered from several limitations. First, the experimental sample size was small. Hence, the process of data collection must be strengthened further to increase the sample size. Second, multicenter studies are needed to analyze whether the relationships identified are valid in other locations; our center is a single-center research institution. Third, we did not perform long-term survival analysis, and further study is needed to identify the long-term effects of sarcopenia on elderly CRC patients. 
In conclusion, it was confirmed that sarcopenia is a risk factor associated with postoperative complications in elderly CRC patients. In addition, hypoalbuminemia was also identified as an undesirable factor markedly associated with elderly patients. We also found that laparoscopy is a protective factor for postoperative complications in CRC patients, especially for colon cancer patients. In summary, the treatment of sarcopenia and hypoproteinemia and the use of intraoperative laparoscopy can reduce the risks associated with CRC surgery.

Acknowledgments: This study was funded by the Department of Health of Zhejiang Province, China (grant no. 2016ZDA017), Huzhou Municipal Science and Technology Bureau (grant no. 2015GY17), and Wenzhou Municipal Science and Technology Bureau (grant no. Y20160408). There are no other commercial interests or sources of financial or material support to declare. The protocol for this study was approved by the ethics committee of The First Affiliated Hospital of Wenzhou Medical University and all participants provided written informed consent.

\section{References}

[1] BRAY F, FERLAY J, SOERJOMATARAM I, SIEGEL RL, TORRE LA et al. Global cancer statistics 2018: GLOBOCAN estimates of incidence and mortality worldwide for 36 cancers in 185 countries. CA Cancer J Clin 2018; 68: 394-424. https://doi.org/10.3322/caac.21492

[2] DODSON S, BARACOS VE, JATOI A, EVANS WJ, CELLA $\mathrm{D}$ et al. Muscle wasting in cancer cachexia: clinical implications, diagnosis, and emerging treatment strategies. Annu Rev Med 2011; 62: 265-279. https://doi.org/10.1146/annurev-med-061509-131248

[3] BECKMANN K, MOORE J, WATTCHOW D, YOUNG G, RODER D. Short-term outcomes after surgical resection for colorectal cancer in South Australia. J Eval Clin Pract 2017; 23: 316-324. https://doi.org/10.1111/jep.12612

[4] HENDREN S, BIRKMEYER JD, YIN H, BANERJEE M, SONNENDAY C et al. Surgical complications are associated with omission of chemotherapy for stage III colorectal cancer. Dis Colon Rectum 2010; 53: 1587-1593. https://doi. org/10.1007/DCR.0b013e3181f2f202

[5] COHEN ME, BILIMORIA KY, KO CY, HALL BL. Development of an American College of Surgeons National Surgery Quality Improvement Program: morbidity and mortality risk calculator for colorectal surgery. J Am Coll Surg 2009; 208: 1009-1016. https://doi.org/10.1016/j.jamcollsurg.2009.01.043

[6] LAW WL, POON JT, FAN JK, LO OS. Survival following laparoscopic versus open resection for colorectal cancer. Int J Colorectal Dis 2012; 27: 1077-1085. https://doi.org/10.1007/ s00384-012-1424-8

[7] SJO OH, LARSEN S, LUNDE OC, NESBAKKEN A. Short term outcome after emergency and elective surgery for colon cancer. Colorectal Dis 2009; 11: 733-739. https://doi. org/10.1111/j.1463-1318.2008.01613.x
[8] KWIATKOWSKI AP, STEPINSKA G, STANOWSKI E, PASNIK K. Implementation of laparoscopic approach in colorectal surgery - a single center's experience. Wideochir Inne Tech Maloinwazyjne 2018; 13: 27-32. https://doi. org/10.5114/wiitm.2018.72748

[9] LANDI F, VALLRIBERA F, RIVERA JP, BERTOLI P, ARMENGOL $\mathrm{M}$ et al. Morbidity after laparoscopic and open rectal cancer surgery: a comparative analysis of morbidity in octogenarians and younger patients. Colorectal Dis 2016; 18: 459-467. https://doi.org/10.1111/codi.13136

[10] COSTA COSTA MD, VIEIRA DE MELO CY, AMORIM AC, CIPRIANO TORRES DDE O, DOS SANTOS AC. Association Between Nutritional Status, Inflammatory Condition, and Prognostic Indexes with Postoperative Complications and Clinical Outcome of Patients with Gastrointestinal Neoplasia. Nutr Cancer 2016; 68: 1108-1114. https://doi.org /10.1080/01635581.2016.1206578

[11] ZHANG WT, LIN J, CHEN WS, HUANG YS, WU RS et al. Sarcopenic Obesity Is Associated with Severe Postoperative Complications in Gastric Cancer Patients Undergoing Gastrectomy: a Prospective Study. J Gastrointest Surg 2018; 22: 1861-1869. https://doi.org/10.1007/s11605-018-3835-5

[12] LIN J, ZHANG W, HUANG Y, CHEN W, WU R et al. Sarcopenia is associated with the neutrophil/lymphocyte and platelet/lymphocyte ratios in operable gastric cancer patients: a prospective study. Cancer Manag Res 2018; 10: 4935-4944. https://doi.org/10.2147/CMAR.S175421

[13] CHEN LK, LIU LK, WOO J, ASSANTACHAI P, AUYEUNG TW et al. Sarcopenia in Asia: consensus report of the Asian Working Group for Sarcopenia. J Am Med Dir Assoc 2014; 15: 95-101. https://doi.org/10.1016/j.jamda.2013.11.025

[14] MARGADANT CC, BRUNS ER, SLOOTHAAK DA, VAN DUIJVENDIJK P, VAN RAAMT AF et al. Lower muscle density is associated with major postoperative complications in older patients after surgery for colorectal cancer. Eur J Surg Oncol 2016; 42: 1654-1659. https://doi.org/10.1016/j. ejso.2016.05.040

[15] HAMIDI M, HO C, ZEESHAN M, O'KEEFFE T, HAMZA A et al. Can Sarcopenia Quantified by Computed Tomography Scan Predict Adverse Outcomes in Emergency General Surgery? J Surg Res 2019; 235: 141-147. https://doi. org/10.1016/j.jss.2018.09.027

[16] MEYER CP, RIOS-DIAZ AJ, DALELA D, RAVI P, SOOD A et al. The association of hypoalbuminemia with early perioperative outcomes - A comprehensive assessment across 16 major procedures. Am J Surg 2017; 214: 871-883. https://doi. org/10.1016/j.amjsurg.2016.11.023

[17] CAGLIA P, TRACIA A, BUFFONE A, AMODEO L, TRACIA L et al. Physiopathology and clinical considerations of laparoscopic surgery in the elderly. Int J Surg 2016; 33: S97S102. https://doi.org/10.1016/j.ijsu.2016.05.044

[18] KANG SC, KIM HI, KIM MG. Low Serum Albumin Level, Male Sex, and Total Gastrectomy Are Risk Factors of Severe Postoperative Complications in Elderly Gastric Cancer Patients. J Gastric Cancer 2016; 16: 43-50. https://doi. org/10.5230/jgc.2016.16.1.43 
[19] KONDRUP J, RASMUSSEN HH, HAMBERG O, STANGA Z; AD HOC ESPEN WORKING GROUP. Nutritional risk screening (NRS 2002): a new method based on an analysis of controlled clinical trials. Clin Nutr 2003; 22: 321-336. https://doi.org/10.1016/s0261-5614(02)00214-5

[20] CLAVIEN PA, BARKUN J, DE OLIVEIRA ML, VAUTHEY JN, DINDO D et al. The Clavien-Dindo classification of surgical complications: five-year experience. Ann Surg 2009; 250: 187-196. https://doi.org/10.1097/SLA.0b013e3181b13ca2

[21] MOURTZAKIS M, PRADO CM, LIEFFERS JR, REIMAN T, MCCARGAR LJ et al. A practical and precise approach to quantification of body composition in cancer patients using computed tomography images acquired during routine care. Appl Physiol Nutr Metab 2008; 33: 997-1006. https:// doi.org/10.1139/H08-075

[22] BOER BC, DE GRAAFF F, BRUSSE-KEIZER M, BOUMAN DE, SLUMP CH et al. Skeletal muscle mass and quality as risk factors for postoperative outcome after open colon resection for cancer. Int J Colorectal Dis 2016; 31: 1117-1124. https://doi.org/10.1007/s00384-016-2538-1

[23] ZHUANG CL, HUANG DD, PANG WY, ZHOU CJ, WANG SL et al. Sarcopenia is an Independent Predictor of Severe Postoperative Complications and Long-Term Survival After Radical Gastrectomy for Gastric Cancer: Analysis from a Large-Scale Cohort. Medicine (Baltimore) 2016; 95: e3164. https://doi.org/10.1097/MD.0000000000003164

[24] CHARLIER R, KNAEPS S, MERTENS E, VAN ROIE E, DELECLUSE $\mathrm{C}$ et al. Age-related decline in muscle mass and muscle function in Flemish Caucasians: a 10-year follow-up. Age (Dordr) 2016; 38: 36. https://doi.org/10.1007/s11357016-9900-7

[25] DODDS RM, ROBERTS HC, COOPER C, SAYER AA. The Epidemiology of Sarcopenia. J Clin Densitom 2015; 18: 461466. https://doi.org/10.1016/j.jocd.2015.04.012

[26] MARUSCH F, KOCH A, SCHMIDT U, STEINERT R, UEBERRUECK $\mathrm{T}$ et al. The impact of the risk factor "age" on the early postoperative results of surgery for colorectal carcinoma and its significance for perioperative management. World J Surg 2005; 29: 1013-1021; discussion 1021-2. https://doi.org/10.1007/s00268-005-7711-6

[27] LANDI F, CRUZ-JENTOFT AJ, LIPEROTI R, RUSSO A, GIOVANNINI $S$ et al. Sarcopenia and mortality risk in frail older persons aged 80 years and older: results from ilSIRENTE study. Age Ageing 2013; 42: 203-209. https://doi. org/10.1093/ageing/afs194

[28] YANG J, ZHANG T, FENG D, DAI X, LV T et al. A new diagnostic index for sarcopenia and its association with short-term postoperative complications in patients undergoing surgery for colorectal cancer. Colorectal Dis 2019; 21: 538-547. https://doi.org/10.1111/codi.14558

[29] TAN BH, FEARON KC. Cachexia: prevalence and impact in medicine. Curr Opin Clin Nutr Metab Care 2008; 11: 400407. https://doi.org/10.1097/MCO.0b013e328300ecc1

[30] PRADO CM, LIEFFERS JR, MCCARGAR LJ, REIMAN T, SAWYER MB et al. Prevalence and clinical implications of sarcopenic obesity in patients with solid tumours of the respiratory and gastrointestinal tracts: a populationbased study. Lancet Oncol 2008; 9: 629-635. https://doi. org/10.1016/S1470-2045(08)70153-0
[31] STRASSER B, STEINDORF K, WISKEMANN J, ULRICH CM. Impact of resistance training in cancer survivors: a meta-analysis. Med Sci Sports Exerc 2013; 45: 2080-2090. https://doi.org/10.1249/MSS.0b013e31829a3b63

[32] MARTONE AM, LATTANZIO F, ABBATECOLA AM, CARPIA DL, TOSATO $\mathrm{M}$ et al. Treating sarcopenia in older and oldest old. Curr Pharm Des 2015; 21: 1715-1722. https:// doi.org/10.2174/1381612821666150130122032

[33] YARLA NS, POLITO A, PELUSO I. Effects of Olive Oil on TNF-alpha and IL-6 in Humans: Implication in Obesity and Frailty. Endocr Metab Immune Disord Drug Targets 2018; 18: 63-74. https://doi.org/10.2174/187153031766617112015 0329

[34] MARZETTI E, CALVANI R, TOSATO M, CESARI M, DI BARI $M$ et al. Sarcopenia: an overview. Aging Clin Exp Res 2017; 29: 11-17. https://doi.org/10.1007/s40520-016-0704-5

[35] HUANG HY, SHI JF, GUO LW, BAI YN, LIAO XZ et al. Expenditure and financial burden for the diagnosis and treatment of colorectal cancer in China: a hospital-based, multicenter, cross-sectional survey. Chin J Cancer 2017; 36: 41. https://doi.org/10.1186/s40880-017-0209-4

[36] HASKINS IN, BAGINSKY M, AMDUR RL, AGARWAL S. Preoperative hypoalbuminemia is associated with worse outcomes in colon cancer patients. Clin Nutr. 2017; 36: 13331338. https://doi.org/10.1016/j.clnu.2016.08.023

[37] HARDT J, PILZ L, MAGDEBURG J, KIENLE P, POST S et al. Preoperative hypoalbuminemia is an independent risk factor for increased high-grade morbidity after elective rectal cancer resection. Int J Colorectal Dis 2017; 32: 1439-1446. https://doi.org/10.1007/s00384-017-2884-7

[38] NAZHA B, MOUSSALY E, ZAAROUR M, WEERASINGHE C, AZAB B. Hypoalbuminemia in colorectal cancer prognosis: Nutritional marker or inflammatory surrogate? World J Gastrointest Surg 2015; 7: 370-377. https://doi. org/10.4240/wjgs.v7.i12.370

[39] CABRERIZO S, CUADRAS D, GOMEZ-BUSTO F, ARTAZA-ARTABE I, MARÍN-CIANCAS F et al. Serum albumin and health in older people: Review and meta analysis. Maturitas 2015; 81: 17-27. https://doi.org/10.1016/j.maturitas.2015.02.009

[40] VOLKERT D, BERNER YN, BERRY E, CEDERHOLM T, COTI BERTRAND $\mathrm{P}$ et al. ESPEN Guidelines on Enteral Nutrition: Geriatrics. Clin Nutr 2006; 25: 330-360. https:// doi.org/10.1016/j.clnu.2006.01.012

[41] GOLDWASSER P, FELDMAN J. Association of serum albumin and mortality risk. J Clin Epidemiol 1997; 50: 693-703. https://doi.org/10.1016/s0895-4356(97)00015-2

[42] SEILER WO. Clinical pictures of malnutrition in ill elderly subjects. Nutrition 2001; 17: 496-498. https://doi. org/10.1016/s0899-9007(01)00558-5

[43] SOETERS PB, WOLFE RR, SHENKIN A. Hypoalbuminemia: Pathogenesis and Clinical Significance. JPEN J Parenter Enteral Nutr 2019; 43: 181-193. https://doi.org/10.1002/ jpen. 1451

[44] GATTA A, VERARDO A, BOLOGNESI M. Hypoalbuminemia. Intern Emerg Med 2012; 7: S193-199. https://doi. org/10.1007/s11739-012-0802-0 
[45] HAN SA, LEE WY, PARK CM, YUN SH, CHUN HK. Comparison of immunologic outcomes of laparoscopic vs open approaches in clinical stage III colorectal cancer. Int J Colorectal Dis 2010; 25: 631-638. https://doi.org/10.1007/ s00384-010-0882-0

[46] FRANCO I, DE'ANGELIS N, CANOUI-POITRINE F, LE ROY B, COURTOT L et al. Feasibility and Safety of Laparoscopic Right Colectomy in Oldest-Old Patients with Colon Cancer: Results of the CLIMHET Study Group. J Laparoendosc Adv Surg Tech A 2018; 28: 1326-1333. https://doi. org/10.1089/lap.2018.0040

[47] FRASSON M, BRAGA M, VIGNALI A, ZULIANI W, DI CARLO V. Benefits of laparoscopic colorectal resection are more pronounced in elderly patients. Dis Colon Rectum 2008; 51: 296-300. https://doi.org/10.1007/s10350-007-9124-0

[48] SUJATHA-BHASKAR S, ALIZADEH RF, INABA CS, KOH CY, JAFARI MD et al. Respiratory complications after colonic procedures in chronic obstructive pulmonary disease: does laparoscopy offer a benefit? Surg Endosc 2018; 32: 1280-1285. https://doi.org/10.1007/s00464-017-5805-5

[49] ZHANG C, DI J, JIANG B, CUI M, WANG Z et al. Prognostic factors for patients with stage II colon cancer: results of a prospective study. Int J Colorectal Dis. 2016; 31(1): 123-9. https://doi.org/10.1007/s00384-015-2401-9

[50] NEKI K, ETO K, KOSUGE M, OHKUMA M, NOAKI R et al. Comparison of Postoperative Outcomes Between Laparoscopic and Open Surgery for Colorectal Cancer. Anticancer Res 2017; 37: 5173-5177. https://doi.org/10.21873/anticanres.11939

[51] BIONDI A, GROSSO G, MISTRETTA A, MARVENTANO S, TOSCANO C et al. Laparoscopic-assisted versus open surgery for colorectal cancer: short- and long-term outcomes comparison. J Laparoendosc Adv Surg Tech A 2013; 23: 1-7. https://doi.org/10.1089/lap.2012.0276
[52] HUANG C, HUANG R, JIANG T, HUANG K, CAO J et al. Laparoscopic and open resection for colorectal cancer: an evaluation of cellular immunity. BMC Gastroenterol 2010; 10: 127. https://doi.org/10.1186/1471-230X-10-127

[53] ZHU Q, MAO Z, JIN J, DENG Y, ZHENG M et al. The safety of $\mathrm{CO} 2$ pneumoperitoneum for elderly patients during laparoscopic colorectal surgery. Surg Laparosc Endosc Percutan Tech 2010; 20: 54-57. https://doi.org/10.1097/ SLE.0b013e3181ce1462

[54] MARTINEZ-PEREZ A, CARRA MC, BRUNETTI F, DE'ANGELIS N. Short-term clinical outcomes of laparoscopic vs open rectal excision for rectal cancer: A systematic review and meta-analysis. World J Gastroenterol 2017; 23: 7906-7916. https://doi.org/10.3748/wjg.v23.i44.7906

[55] ATHANASIOU CD, ROBINSON J, YIASEMIDOU M, LOCKWOOD S, MARKIDES GA. Laparoscopic vs open approach for transverse colon cancer. A systematic review and meta-analysis of short and long term outcomes. Int J Surg 2017; 41: 78-85. https://doi.org/10.1016/j. ijsu.2017.03.050

[56] MUKAI T, AKIYOSHI T, UENO M, FUKUNAGA Y, NAGAYAMA $S$ et al. Outcomes of laparoscopic surgery for colorectal cancer in oldest-old patients. Surg Laparosc Endosc Percutan Tech 2014; 24: 366-369. https://doi. org/10.1097/SLE.0b013e31829012ca

[57] KESKIN M, AKICI M, AGCAOGLU O, YEGEN G, SAGLAM E et al. Open Versus Laparoscopic Surgery for Rectal Cancer: Single-Center Results of 587 Cases. Surg Laparosc Endosc Percutan Tech 2016; 26: e62-8. https://doi. org/10.1097/SLE.0000000000000267 